International Journal of Pure and Applied Mathematics

Volume 111 No. 2 2016, 209-217

ISSN: 1311-8080 (printed version); ISSN: 1314-3395 (on-line version)

url: http://www.ijpam.eu

doi: $10.12732 /$ ijpam.v111i2.7

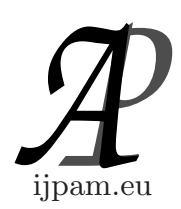

\title{
STRENGTHENED MICHAEL'S THEOREM AND ITS APPLICATIONS TO MAPS AND SPACES
}

\author{
Nigar Tuncer Özarslan \\ Bahcesehir University \\ Istanbul, TURKEY
}

\begin{abstract}
In this paper it is provided a globalization theorem which strengthens a theorem of Michael for local properties of the spaces and maps. Applications include a globalization theorem for numerability for spaces, metrizability and some new proofs for known globalization theorems for SEP, including the Uniformization Theorem of Hurewicz.
\end{abstract}

AMS Subject Classification: 54F99, 55R05

Key Words: uniformization, metrization

\section{Introduction}

A topological space $X$ has property $\mathcal{P}$ locally if and only if every point of $X$ has a neighborhood with the property $\mathcal{P}$ and in this case it is said that $\mathcal{P}$ is local property of $X$. If the space itself has property $\mathcal{P}$ then it is called a global property of $X$ or $X$ has property $\mathcal{P}$ globally. One can similarly define local and global for properties of mappings of one space to another. Since any space is a neighborhood of each of its points, it is obvious that if a space $X$ has a certain property globally then it has that property locally ${ }^{1}$. Some few properties also

$\begin{array}{lr}\text { Received: } & \text { August 5, } 2016 \\ \text { Revised: } & \text { October 30, } 2016 \\ \text { Published: } & \text { December 11, } 2016\end{array}$

(C) 2016 Academic Publications, Ltd. url: www.acadpubl.eu 
satisfies the converse, for example, a local Serre Fibration is a Serre Fibration. But it is clearly not true for many topological properties such as contractibility and metrizability. Most of the time some added conditions are needed in order to get local implies global results. It is natural to raise the question:

Under what conditions is a local property, a global property?

The theorems which answer this question are called globalization theorems. W. Hurewicz [5] proved a globalization theorem which he called Uniformization Theorem for what are now known as Hurewicz Fibrations by putting the paracompactness condition on the base space and Dold similiarly [1] proved a globalization theorem for mapping having Section Extension Property. Nagata and Smirnov proved that a paracompact locally metrizable space is metrizable. Dyer and Eilenberg [3], give such theorems for Inversible Fibrations and Hurewicz Fibrations. These are examples of globalization theorems for special properties. E. Michael [7] answered the above question by showing that an axiomatic treatment can be applied to and introduced G-hereditary and F-hereditary properties. Roughly, he proved that if a space can be covered by a collection of subsets, satisfying certain conditions, each of which has the property, then the space has the property.

The theorem refered to as Michael's Theorem is as follows:

Theorem 1. : Let $X$ be a topological space and $\mathcal{P}$ a property which is $G$-hereditary for $X$. Then, if $X$ is paracompact and if $X$ has property $\mathcal{P}$ locally, then $X$ has property $\mathcal{P}$.

\section{Strengthened Michael's Theorem}

Now we proceed to obtain the strengthened theorem. ${ }^{2}$ We introduce the term numerable sets and NG-hereditary.

Definition 2. For a topological space $X$ and $A \subseteq X, \mathrm{~A}$ is said to be numerable in $X$ if and only if there exists a continuous map $\rho: X \rightarrow[0,1]$ such that $\rho^{-1}(0,1]=A$. In this case $\rho$ is called a numeration of $A$ in $X$.

This definition coincides with the co-zero sets in the literature but we prefer to use in order to be consistent with other terminology we use for spaces. It is obvious that every open set is numerable in a metric space. Now we give the definition of NG-hereditary property.

\footnotetext{
${ }^{1}$ For some important properties, the word local is used with different meaning from that given here. For example, there there are spaces which are connected but not locally connected.

${ }^{2}$ Nagami Neiô in [8] gives similiar strengthening theorem without any proofs
} 
Definition 3. Let $\mathcal{P}$ be a property and $X$ be a topological space. $\mathcal{P}$ is called $N G$-hereditary for $X$ if the following conditions are satisfied:

i) If subspace $Y$ of $X$ has property $\mathcal{P}$ then every numerable subset of $Y$ has property $\mathcal{P}$.

ii) If $Y$ is the union of two numerable subsets of $X$ both of which have property $\mathcal{P}$, then $Y$ has property $\mathcal{P}$.

iii) If $\left\{U_{\alpha} \mid \alpha \in \Lambda\right\}$ is a discrete collection of sets numerable in $X$ each of which has property $\mathcal{P}$, then $\bigcup_{\alpha \in \Lambda} U_{\alpha}$ has property $\mathcal{P}$.

In Michael's definition of G-hereditary, open sets are used instead of numerable. We state the strengthened Michael's theorem which has essentially the same proof.

Theorem 4. (Strengthened Michael's Theorem): Let $X$ be a space and $\mathcal{P}$ a local property for $X$ which is NG-hereditary. If $X$ is paracompact then $X$ has the property.

\section{Applications to Properties of Spaces}

In this section it is shown that certain properties of spaces are $N G$-hereditary. These facts are used to obtain proofs for globalization theorems.

Definition 5. A topological space $X$ is said to be numerablespace if and only if every open set in $X$ is numerable.

We state the following theorem without any proof which will give new proof some known results about metrizability.

Theorem 6. For any space $X$ being regular, having a $\sigma$-locally finite basis and being a numerable space are $N G$ - hereditary properties for $X$.

The following globalization theorems are concequence of Theorem 6 and the Strengthened Michael's Theorem.

Theorem 7. If the space $X$ is paracompact and locally regular, then $X$ is regular.

Theorem 8. If the space $X$ is paracompact and locally has a $\sigma$-locally finite basis, then $X$ has a $\sigma$-locally finite basis.

Theorem 9. If a space $X$ is paracompact and locally a numerable space then it is numerable.

Furthermore using the characterization of metrizable spaces provided by 
Nagata-Smirnov and stated below, we obtain a new proof for a well known globalization for metrizability.

Theorem 10 (Nagata-Smirnov ). A space is metrizable if and only if it is regular and has $\sigma$ - locally finite basis.

Theorem 11. If $X$ is paracompact and locally metrizable then it is metrizable.

Proof. Since $X$ is locally metrizable, it is locally regular and locally has $\sigma$ - locally finite basis. By Theorem 6 these are NG-hereditary properties, so by Theorem $4, X$ is regular and has $\sigma$-locally finite basis. Therefore it is metrizable.

\section{Applications to Maps}

In this section two types of maps are dealt with, maps having the Section Extension Property (SEP) as defined by Dold in [1] and Hurewicz Fibrations. In each case a certain property is shown to be $N G$-hereditary for appropriate spaces. As a result we will obtain proofs for globalization theorems.

\section{Concerning SEP}

Definition 12. Let $A$ be a subset of a space $X$. A subset $V$ is called a halo around $A$ if and only if there exists a continuous map $\rho: X \rightarrow[0,1]$ such that $A \subseteq \rho^{-1}(1)$ and $V^{c} \subseteq \rho^{-1}(0)$.

Definition 13. Let $\mathcal{F}=(E, p, B)$ be a space over $B$. $\mathcal{F}$ is said to have Section Extension Property (SEP) if and only if for every $A \subseteq B, V$ a halo around $A$ and $s$ a section on $A$ which can be extended to $V$, there is a section $S$ over $B$ such that $S \mid A=s$.

Definition 14. If $\mathcal{F}=(E, p, B)$ is a space over $B$ and $A \subseteq B$, we will say $A$ has property SEP if and only if $\mathcal{F} \mid A=\left(p^{-1}(A), p \mid p^{-1}(A), A\right)$ has SEP.

The following theorem shows that property SEP is NG-hereditary for $B$. With this one sees immediately that the Section Extension Theorem of Dold, the Globalization Theorem for the SEP, is a consequence of the Theorem 6 and the Strengthened Michael's Theorem.

Theorem 15. If $\mathcal{F}=(E, p, B)$ is a space over $B$, then property SEP (as defined above) is NG-hereditary for $B$. 
Proof. In order to prove this we need to show that; i)numerable subsets of subspaces with SEP has SEP, ii)union of two numerable subsets with SEP has SEP, iii)union of discrete collection of numerable subsets with SEP has SEP. Condition i) holds;

Dold proved in $[1]$ (Proposition 2.6.) that if $\mathcal{F}$ has SEP then $\mathcal{F} \mid A$ has SEP for numerable subsets of $B$.

Condition ii) holds;

Let $U_{1}$ and $U_{2}$ be two numerable subsets of $B$ with property SEP. Let $A$ be a subset of $U_{1} \cup U_{2}$ which has a halo with haloing function $\tau: U_{1} \cup U_{2} \rightarrow[0,1]$. And let $\rho_{1}$ and $\rho_{2}$ be numerations of $U_{1}$ and $U_{2}$ respectively.

Assume that $s$ is a section on $\tau^{-1}(1)$ which can be extended to $\tau^{-1}(0,1]$. Take $s_{1}=\left.s\right|_{\tau^{-1}\left[\frac{1}{2}, 1\right] \cap U_{1}}$, then it will be a section on $\tau^{-1}\left[\frac{1}{2}, 1\right] \cap U_{1}$ which can be extended to $V \cap U_{1}$. Since $U_{1}$ has SEP, this can be extended to a section on $U_{1}$, namely $S_{1}$.

Now define a map $\phi: U_{1} \cup U_{2} \rightarrow[0,1]$ by

$$
\phi(b)= \begin{cases}1, & \text { if } \rho_{1}(b) \geq \rho_{2}(b) \\ \max \left\{\tau(b), \frac{\rho_{1}(b)}{\rho_{2}(b)}\right\}, & \text { if } \rho_{1}(b) \leq \rho_{2}(b)\end{cases}
$$

The following facts about $\phi$ will be needed;

1. $\tau^{-1}(1) \subseteq \phi^{-1}(1)$.

2. $\phi\left|U_{2}-U_{1}=\tau\right| U_{2}-U_{1}$ and so $\phi^{-1}\left(\frac{3}{4}, 1\right] \cap U_{2} \subseteq U_{1} \cup \tau^{-1}\left[\frac{1}{2}, 1\right]$.

3. If $\rho_{1}(b)=\rho_{2}(b)$, then $b \in U_{1} \cap U_{2}$ and $\phi(b)=1$.

Since $S_{1}$ is defined on $U_{1}$ and agrees with $s$ on $\tau^{-1}\left[\frac{1}{2}, 1\right]$, we can define a section on $U_{1} \cup \tau^{-1}\left[\frac{1}{2}, 1\right]$ by

$$
S_{1}^{\prime}(b)= \begin{cases}s(b), & \text { if } \tau(b) \geq \frac{1}{2} \\ S_{1}(b), & \text { if } \tau(b) \leq \frac{1}{2}\end{cases}
$$

Let's take section $S_{1}^{\prime} \mid \phi^{-1}(1) \cap U_{2}$. Then $S_{1}^{\prime}$ has an extention to $\phi^{-1}\left(\frac{3}{4}, 1\right] \cap U_{2}$, which is a halo around $\left.\phi^{-1}(1) \cap U_{2}\right)$. Since $U_{2}$ has SEP, $S_{1}^{\prime} \mid \phi^{-1}(1) \cap U_{2}$ has an extention $S_{2}$ to $U_{2}$. Obviously, $S_{2}\left|\tau^{-1}(1) \cap U_{2}=s\right| \tau^{-1}(1) \cap U_{2}$.

Finally, we define a section on $U_{1} \cup U_{2}$ by,

$$
S= \begin{cases}S_{1}, & \text { if } \rho_{1}(b) \geq \rho_{2}(b) \\ S_{2}, & \text { if } \rho_{1}(b) \leq \rho_{2}(b)\end{cases}
$$

then $S \mid \tau^{-1}(1)=s$, because $S_{1}\left|\tau^{-1}(1)=S_{2}\right| \tau^{-1}(1)=s$. 
It is continuous, because $S_{1}$ and $S_{2}$ are continuous on $U_{1}$ and $U_{2}$ respectively and $S_{1}(b)=S_{2}(b)$ when $\rho_{1}(b)=\rho_{2}(b)$.

iii) holds;

Let $U=\sqcup_{\alpha \in \Lambda} U_{\alpha}$, where $\left\{U_{\alpha}\right\}$ is a discrete collection of subsets of $X$ with property SEP. Let $O$ be a subset of $U$ and $V$ a halo around it with haloing function $\rho, s$ a section on $O$ which can be extended to $V$. Since each $\mathcal{F} \mid U_{\alpha}$ has SEP and $V \cap U_{\alpha}$ is a halo around $O \cap U_{\alpha}, s \mid O \cap U_{\alpha}$ can be extended to $U_{\alpha}$, say $s_{\alpha}$ for all $\alpha$. Now, define

$$
S: U \rightarrow E \quad \text { as } \quad S(b)=s_{\alpha}(b), b \in U_{\alpha}
$$

We are now able to provide a new proof for the SEP Globalization Theorem.

Theorem 16 (Section Extension Theorem). ([3] 2.7.) Let $p: E \rightarrow B$ be space over $B$. If there exists a numerable cover (in the sense of Dold, same as normal covers) $\left\{V_{\lambda}\right\}_{\lambda \in \Lambda}$ of $B$ such that $p$ has the property SEP over each $V_{\lambda}$, then $p$ has the property SEP.

Proof. By Theorem 6 and Theorem 15 result follows.

\section{Concerning Hurewicz Fibration}

We give a new proof of Uniformization Theorem of Hurewicz. First we provide definitions of Hurewicz and some details concerning his proof written using our notation and language.

Uniformization Theorem A (regular) local Hurewicz Fibration $\mathcal{F}=$ $(E, p, B)$ is a (regular) Hurewicz Fibration provided $B$ is paracompact.

Hurewicz proves the following theorem from which the Uniformization Theorem follows.

Theorem ([5], 5.1.) If $\mathcal{F}=(E, p, B)$ and $B$ has a normal cover $\left\{U_{\alpha}\right\}$ such that for every $\alpha, \mathcal{F} \mid U_{\alpha}$ is a (regular) Hurewicz Fibration then $\mathcal{F}$ is a (regular) Hurewicz Fibration.

To prove this Hurewicz shows that his hypothesis yields a normal cover of $B^{I}$ each element of which is liftable. While few details for this are given, they are easily provided. The completion of his proof depends on the following theorem for which the ommitted details are not so easily provided. Here we show that being liftable is NG-hereditary for $B^{I}$ and thus obtain a proof for Theorem ([5] 5.4) and consequently a new proof for The Uniformization Theorem.

Theorem $([5], 5.4)$ If $\mathcal{F}=(E, p, B)$ is a space over $B$ and $B^{I}$ has a normal cover $\left\{W_{\mu}\right\}$ such that each $W_{\mu}$ is liftable, then $B^{I}$ is liftable. 
This could be called the key ingredient of Hurewicz proof and it has few details. It will be shown now that this theorem is a consequence of Theorem 4 .

Definition 17. Let $p: E \rightarrow B$ be a map. Given a subset $W \in B^{I}$, let $\widehat{W}$ be the set of all triples $(e, \omega, s)$ with $e \in E, \omega \in W^{I} 0 \leq s \leq 1, \omega(s)=p(e)$. By an extended lifting function over $\widehat{W}$ is a meant a map

$$
\Lambda: \widehat{W} \rightarrow E^{I}
$$

satisfying the conditions

$$
p \Lambda(e, \omega, s)=\omega, \quad \Lambda(e, \omega, s)(s)=e
$$

for every triple in $\widehat{W}$. The set $W$ will be called liftable if an extended lifting function can be defined over $\widehat{W}$.

Theorem 18. Let $p: E \rightarrow B$ be a map. Being liftable is an NG-hereditary for $B^{I}$.

Proof. Condition i) holds;

Let $Y$ be a subspace of $B^{I}$ which is liftable and $W$ a numerable subset of $Y$. Let $\Lambda: \widehat{Y} \rightarrow E^{I}$ be an extended lifting function over $Y$ and note that $\widehat{W} \subseteq \widehat{Y}$. Define a map on $\widehat{W}$ as

$$
\Lambda^{\prime}=\Lambda \mid \widehat{W}
$$

$\Lambda^{\prime}$ is an extended lifting function on $\widehat{W}$. So, numerable subsets of liftable subspaces are liftable.

Condition ii) holds;

Let $W_{1}$ and $W_{2}$ be two numerable and liftable subsets of $B^{I}$, with partition of unity $\rho_{1}$ and $\rho_{2}$ obtained by their numerations as the way described in Proposition 2.3.1. Let $\Lambda_{1}$ and $\Lambda_{2}$ lifting functions over $W_{1}$ and $W_{2}$, respectively.

Define maps $f_{1}$ and $f_{2}, f_{i}: \mathrm{I} \times \rho_{1}^{-1}\left[\frac{1}{4}, \frac{3}{4}\right] \rightarrow \mathrm{I}$ by

$$
\begin{gathered}
f_{1}(s, \omega)=\frac{s}{2}\left(3-4 \rho_{1}(\omega)\right) \\
f_{2}(s, \omega)=s+\left(\frac{1-s}{2}\right)\left(4 \rho_{1}(\omega)-1\right)
\end{gathered}
$$

Note that

1. $0 \leq f_{1}(s, \omega) \leq s \leq f_{2}(s, \omega) \leq 1$ for all $(s, \omega)$ 
2. If $\rho_{1}(\omega)=\frac{1}{4}$ then $f_{1}(s, \omega)=f_{2}(s, \omega)=s$ and if $\rho_{1}(\omega)=\frac{3}{4}$ then $f_{1}(s, \omega)=$ 0 and $f_{2}(s, \omega)=1$.

Define a map on ${\widehat{W_{1} \cup W_{2}}}_{\text {by }}$

$$
\Lambda(e, \omega, s)(t)= \begin{cases}\Lambda_{2}(e, \omega, s)(t), & \text { if } \quad 0 \leq \rho_{1}(\omega) \leq \frac{1}{4} \\ \Lambda^{\prime}(e, \omega, s)(t), & \text { if } \quad \frac{1}{4} \leq \rho_{1}(\omega) \leq \frac{3}{4} \\ \Lambda_{1}(e, \omega, s)(t), & \text { if } \quad \frac{3}{4} \leq \rho_{1}(\omega) \leq 1\end{cases}
$$

where

$$
\Lambda^{\prime}(e, \omega, s)=\left\{\begin{array}{lc}
\Lambda_{2}\left(\Lambda_{1}(e, \omega, s)\left(f_{1}(s, \omega)\right), \omega, f_{1}(s, \omega)\right)(t), & t \leq f_{1}(s, \omega) \\
\Lambda_{1}(e, \omega, s)(t), & f_{1}(s, \omega) \leq t \leq f_{2}(s, \omega) \\
\Lambda_{2}\left(\Lambda_{1}(e, \omega, s)\left(f_{2}(s, \omega)\right), \omega, f_{2}(s, \omega)\right)(t), & f_{2}(s, \omega) \leq t
\end{array}\right.
$$

$\Lambda^{\prime}$ and then $\Lambda$ are defined on various closed sets and are continuous on these by continuity of the $f_{i}$ 's and $\Lambda_{i}$ 's. Continuity of $\Lambda$ follows from the fact that they agree on the intersections of the closed sets.

Condition iii) holds;

Let $\left\{W_{\alpha}\right\}$ be a discrete collection of liftable subsets of $B^{I} . \Lambda(e, \omega, s)(t)=$ $\Lambda_{\alpha}(e, \omega, s)(t), \quad(e, \omega, s) \in \widehat{U}_{\alpha}$ is an extended lifting function on $\widehat{\cup_{\alpha} U_{\alpha}}$.

\section{Acknowledgments and Remarks}

The results of this work are contained in a thesis submitted to the faculty of Binghamton University State University of New York in partial fulfillment of the requirements for Ph. D. degree, in August, 2008.

\section{References}

[1] A. Dold, Partitions of unity in the theory of fibration, Annals of Mathematics, 78 (1963), 223-265, doi: 10.2307/1970341.

[2] V. Dugundji, Topology, Allyn and Bacon Inc., Boston, 1966.

[3] E. Dyer, S. Eilenberg, Globalizing fibrations with schedules, Fund. Math., 130, No. 2 (1988), 125-136.

[4] S. Eilenberg, N. Steenrod, Foundations of Algebraic Topology, Priceton Univ. Press, 1952.

[5] W. Hurewicz, On the concept of fiber spaces, Proc. Nat. Acad. Sci. USA, 41 (1955), 956-961, doi: 10.1073/pnas.41.11.956.

[6] W. Huebsch, Covering homotopy, Duke Math. Journal (1956), 281-191, doi: 10.1215/S0012-7094-56-02326-2. 
[7] E. Michael, Local properties of topological spaces, Duke Math. Journal, 21 (1954), doi: S0012-7094-54-02117-1.

[8] K. Nagami, Local properties of topological spaces, Proc. Japan Acad., 32 (1956), 320-322, doi: $10.3792 / \mathrm{psa} / 1195525373$. 
